Original paper

\title{
Evaluation of thyroid function and thyroid autoimmune disease in patients with non-alcoholic fatty liver disease
}

\author{
Rania Naguib ${ }^{1,2}$, Amel Fayed ${ }^{1}$, Eman Z. Elkemary ${ }^{3}$, Hend Naguib ${ }^{4}$ \\ ${ }^{1}$ Clinical Science Department, College of Medicine, Princess Nourah bint Abdulrahman University, Riyadh, KSA \\ 2Internal Medicine Department, Endocrinology Unit, Faculty of Medicine, Alexandria University, Alexandria, Egypt \\ ${ }^{3}$ Clinical Pathology Department, Faculty of Medicine, Alexandria University, Alexandria, Egypt \\ ${ }^{4}$ Internal Medicine Department, Hepatology Unit, Faculty of Medicine, Alexandria University, Alexandria, Egypt
}

\begin{abstract}
Aim of the study: Evaluation of thyroid function and thyroid autoimmunity in patients with non-alcoholic fatty liver disease (NAFLD).

Material and methods: A case control study. Fifty patients with NAFLD and 50 control subjects matched by gender and age were recruited. Serum thyroid stimulating hormone (TSH) and free thyroxine (FT4) were measured to assess thyroid function. Thyroid autoimmune disease was evaluated by measuring thyroid peroxidase antibody (TPOAb) and anti-thyroglobulin antibodies (TgAb). The FIB-4 score and the APRI score were calculated to assess the degree of fibrosis. The association between thyroid parameters and NAFLD was explored.

Results: About one quarter of patients with NAFLD had hypothyroidism compared to $10 \%$ of the control group whilst $6 \%$ of NAFLD patients had hyperthyroidism compared to $2 \%$ of the controls. NAFLD cases showed substantially higher TSH and lower F4 compared to controls; meanwhile, levels of fibrosis indices (FIB-4 and APRI score) were significantly higher among hypothyroid patients in both cases and controls. TSH had a positive strong correlation with FIB-4 and APRI score, whereas FT4 had a negative significant correlation with both fibrosis indicators, and this clinical relationship was similar in NAFLD cases and controls.

Conclusions: Hypothyroidism is more prevalent among patients with NAFLD compared to controls and high levels of TSH with low FT4 might be a risk factor for NAFLD and may impact the development of liver fibrosis. The role of thyroid autoimmunity in NAFLD needs further assessment. NAFLD patients should be monitored by yearly TSH and FT4 testing.
\end{abstract}

Key words: thyroid function, liver fibrosis, non-alcoholic fatty liver disease, thyroid autoimmunity.

\section{Address for correspondence}

Dr. Rania Naguib, MD, PhD, FRCP, Clinical Science Department, College of Medicine, Princess Nourah bint Abdulrahman University, Riyadh, KSA; Internal Medicine Department, Endocrinology Unit, Faculty of Medicine, Alexandria University, Alexandria, Egypt, e-mail: ranianaguib2000@yahoo.com

\section{Introduction}

Non-alcoholic fatty liver disease (NAFLD) is one of the most common chronic liver diseases. The disease spectrum includes benign steatosis (fat accumulation in liver cells) and steatohepatitis. A small percentage of NAFLD patients (15\%) who develop steatohepatitis have a significant risk of developing fibrosis in a short period of time which can progress to cirrhosis and hepatocellular carcinoma $[1,2]$. The highest prevalence of NAFLD is 32\% is reported in the Middle East and 31\% in South America with Asia (27\%), the United States (24\%), Europe (23\%), and Africa trailing behind (14\%) [3-5]. Although the pathogenesis of NAFLD is not fully understood, it is widely accepted that NAFLD is caused by the interaction of the complex genetic background 
and environmental factors. Insulin resistance increases adipose tissue lipolysis, leading to free fatty acid and triglyceride deposition in the liver [3]. Metabolic disorders such as hypertension, diabetes, hyperlipidemia, and central obesity are considered risk factors for NAFLD [6,7]. Patients with NAFLD have higher liver-related morbidity or mortality than the general population as well as a high risk of extra-hepatic disorders such as cardiovascular disease, chronic renal disease, and endocrine disorders (i.e., type 2 diabetes mellitus, insulin resistance, metabolic syndrome and thyroid dysfunction) $[5,8]$. Thus, the identification of NAFLD risk factors is required for the development of new screening tools and preventative or treatment strategies. Thyroid hormones are involved in regulating body weight, glucose and lipid metabolism as well as insulin resistance. As a result, thyroid hormones may have a role in the pathogenesis of NAFLD [9]. Hypothyroidism is associated with reduced lipolysis and hepatic uptake of free fatty acids derived from triglycerides $[1,10]$. Thyroid hormone disturbances can lead to hyperlipidemia and obesity, which can contribute to NAFLD [11]. In animal models, thyroid hormone analogues $[12,13]$ were utilized to decrease liver fat accumulation. Thyroid hormone analogues have also been shown in clinical investigations to improve NAFLD [6]. Treatment of hypothyroidism may minimize the risk of NAFLD and associated consequences [11]. Several studies have found an inverse relationship between NAFLD morbidity and thyroid hormone levels in hypothyroid and euthyroid people; thus early identification of atrisk patients is crucial $[6,12,14]$. A link between thyroid disorders and NAFLD is increasingly being addressed and the results are controversial $[11,15,16]$. Thyroid stimulating hormone (TSH) level has been linked to the prevalence of NAFLD in several studies, and TSH level may be an important risk factor for NAFLD [17]. Other thyroid hormones, such free thyroxine (FT4), have been linked to NAFLD $[18,19]$. However, the relationship between NAFLD and FT4 levels varies by population $[1,4]$. The link between thyroid antibodies and NAFLD is unknown, and there have been few previous studies $[16,20]$. It is also unknown whether disturbance in thyroid function influences the risk of fibrosis in NAFLD patients. To validate and better define the suggested link between NAFLD and thyroid function, this study was conducted aiming to estimate the prevalence of thyroid disorders in patients with NAFLD and to correlate thyroid function and thyroid autoimmunity with NAFLD and liver fibrosis scores.

\section{Material and methods}

A case control study. Ethical approval was obtained from the institutional ethics committee and informed consent was obtained from all patients and controls who participated in the study. The study included 50 patients with NAFLD aged 18 years and up who had NAFLD and were evaluated in the Hepatology Outpatient Clinics in Alexandria University Hospitals. Fifty age- and sex-matched participants served as the control group. Controls were required to have normal liver tests [alanine aminotransferase (ALT) $\leq 45 \mathrm{IU} / \mathrm{l}$, aspartate aminotransferase $(\mathrm{AST}) \leq 40 \mathrm{IU} / \mathrm{l}$, bilirubin $\leq 1.5 \mathrm{mg} / \mathrm{dl}$, and alkaline phosphatase $\leq 150 \mathrm{IU} / \mathrm{l})]$, as well as the absence of any acute or chronic liver illness and the absence of fatty liver on at least one radiographic imaging scan. NAFLD was defined as a persistent increase in serum ALT or AST of at least 1.5 times the upper limits of normal for at least 3 months and/or the presence of the following four ultrasonographic criteria: hepatorenal echo contrast, liver brightness, deep attenuation, and vascular blurring in the absence of (a) seropositivity for hepatitis B surface antigen or antibody to hepatitis C virus; (b) alcohol consumption; (c) history of other causes of liver disease; and (d) medications known to produce fatty liver disease during the 6 months before the study and/or a liver biopsy showing more than $10 \%$ steatosis with necro-inflammatory changes after other causes of liver disease had been ruled out [21,22].

Subjects with a history of drinking alcohol, any laboratory or clinical evidence of a coexisting chronic liver disease, such as viral hepatitis, autoimmune hepatitis, hepatobiliary malignancies, Wilson's disease, hemochromatosis, al antitrypsin disease, or chronic cholestatic liver disease, were excluded. Patients with chronic kidney disease, diabetes, metabolic syndrome or a history of surgery that leads to secondary NAFLD such as gastropexy, jejunal bypass, small bowel resection and biliopancreatic diversion were excluded from the study. Patients with current or previous use of fatty liver inducing pharmacological agents such as amiodarone, tamoxifen, corticosteroids, and methotrexate, patients with a history of thyroid disease, previous or current intake of iodine, antithyroid agents or thyroid hormones were also excluded. Pregnancy, breast feeding, use of oral contraceptives or hormonal intrauterine devices were also considered as exclusion criteria.

History taking and physical examination were performed in all participants. The height and weight of the participants were measured. Body mass index (BMI) was calculated as body weight in $\mathrm{kg}$ divided by height in meters squared. Platelet count was performed by 
a Sysmex-XT 1800i automated hematology analyzer (Sysmex, Japan). Laboratory tests such as ALT, AST, total cholesterol, triglycerides (TG) and low-density lipoprotein (LDL) were measured by a Dimension Rxl Max chemistry auto-analyzer (Siemens Health Care diagnostics, USA). Serum thyroid stimulating hormone and FT4 were measured using an ADVIA Centaur XP automated immunoassay analyzer (Siemens Health Care diagnostics, USA). Thyroid peroxidase antibody $(\mathrm{TPOAb})$ and anti-thyroglobulin antibodies (TgAb) were measured using a COBAS E411 automated immunoassay analyzer (Roche Diagnostics, Germany) [23]. TSH (0.4-4.0 mIU/l) and FT4 (0.85-1.95 ng/dl) reference ranges were established. If serum TSH was within the reference range, thyroid function was considered euthyroid. TSH values more than $4.0 \mathrm{mIU} / \mathrm{l}$ alone or together with FT4 levels less than $0.85 \mathrm{ng} / \mathrm{dl}$ were used to diagnose hypothyroidism. TSH values of less than $0.4 \mathrm{mIU} / \mathrm{l}$ and FT4 levels of more than $1.95 \mathrm{ng} / \mathrm{dl}$ were used to characterize hyperthyroidism. TPOAb and $\mathrm{TgAb}$ were considered positive following the directions of the test manufacturer [16]. The FIB-4 score (fibrosis index based on a 4 -factor score) was automatically computed using the formula: age (years) $\times$ AST [U/l]/ (platelets $\left[10^{9} / 1\right] \times(\text { ALT }[\mathrm{U} / 1])^{1 / 2}$, with the patient's age being the age at the time of the research. The APRI score (aspartate aminotransferase to platelet ratio index) was obtained by multiplying "AST/upper limit of normal/platelet count $\left[10^{9} / 1\right]$ ” by 100 . In our institution, the maximum limit of normal for AST is 40 IU/1 [24]. The prevalence of thyroid dysfunction was estimated among NAFLD patients and compared to control subjects. The association between thyroid parameters and NAFLD and the degree of fibrosis was explored.

\section{Statistical analysis of the data}

Data analysis was conducted using IBM SPSS software package version 20.0. (Armonk, NY: IBM Corp). The Kolmogorov-Smirnov test was used to verify the normality of distribution of variables. Comparisons between groups for categorical variables were assessed using the $\chi^{2}$ test or Fisher's exact test accordingly. Student's $t$-test was used to compare two groups for normally distributed quantitative variables while the Mann-Whitney test was used to compare between two groups for non-normally distributed quantitative variables. Spearman's coefficient was used to correlate between quantitative variables. Linear regression models were developed to test the difference between NAFLD cases and controls in different parameters after adjustment for age, BMI and thyroid status. Significance of the obtained results was judged at the $5 \%$ level.

\section{Results}

Both groups were recruited with a similar percentage of males and females, but there was a significant difference in age, as cases were significantly older than controls ( $47.9 \pm 3$ vs. $45.7 \pm 4$ years), and in BMI where cases had higher BMI $\left(31.6 \pm 4.7\right.$ vs. $\left.25.4 \pm 2.3 \mathrm{~kg} / \mathrm{m}^{2}\right)$. As compared to controls, cases showed significantly higher levels of TC, TG, LDL, ALT and AST while the platelet counts were significantly lower $(207.1 \pm 109.7$ vs. 294.6 \pm 71.6 ) (Table 1).

In terms of the thyroid profile, about one quarter of patients with NAFLD had hypothyroidism compared to $10 \%$ of the control group whilst $6 \%$ of NAFLD patients had hyperthyroidism compared to $2 \%$ of the controls $(p<0.01)$ (Table 1$)$.

NAFLD cases showed substantially higher TSH and lower FT4 compared to controls, while levels of fibrosis indices (FIB-4 and APRI score) were significantly higher among hypothyroid patients in both cases and controls (Fig. 1).

Figure 2 and Table 2 illustrate the correlations between TSH and FT4 and fibrosis parameters in NAFLD cases and control. TSH had a positive strong correlation with FIB-4 and APRI score, whereas FT4 had a negative significant correlation with both fibrosis indicators, and this clinical relationship was similar in NAFLD cases and controls.

After adjusting for age, BMI, and thyroid status using regression models, the differences in TSH level, FIB-4, and APRI score between NAFLD cases and controls remained statistically significant. When compared to controls, NAFLD cases would have greater TSH (an average of $0.84 \mu \mathrm{IU} / \mathrm{ml}$ ), higher FIB-4 (one point on average), and APRI score ( 0.45 points on average). However, other differences in FT4, TPOAb and $\mathrm{TgAb}$ did not show any significant differences between NAFLD cases and controls after adjustment for age, BMI and thyroid status.

\section{Discussion}

The present study demonstrated that the prevalence of hypothyroidism was significantly higher in patients with NAFLD than in controls and the TSH level was significantly higher while FT4 was significantly lower in patients with NAFLD. Furthermore, the FIB-4 and APRI scores were significantly higher in patients with hypothyroidism than in those with euthyroidism. High TSH and low FT4 are associated with liver fibrosis. Thyroid hormones are powerful regulators of metabolic and energy balance, and they have been linked to a variety of metabolic disorders. Hypothyroidism 
Table 1. Comparison between the two studied groups according to different parameters

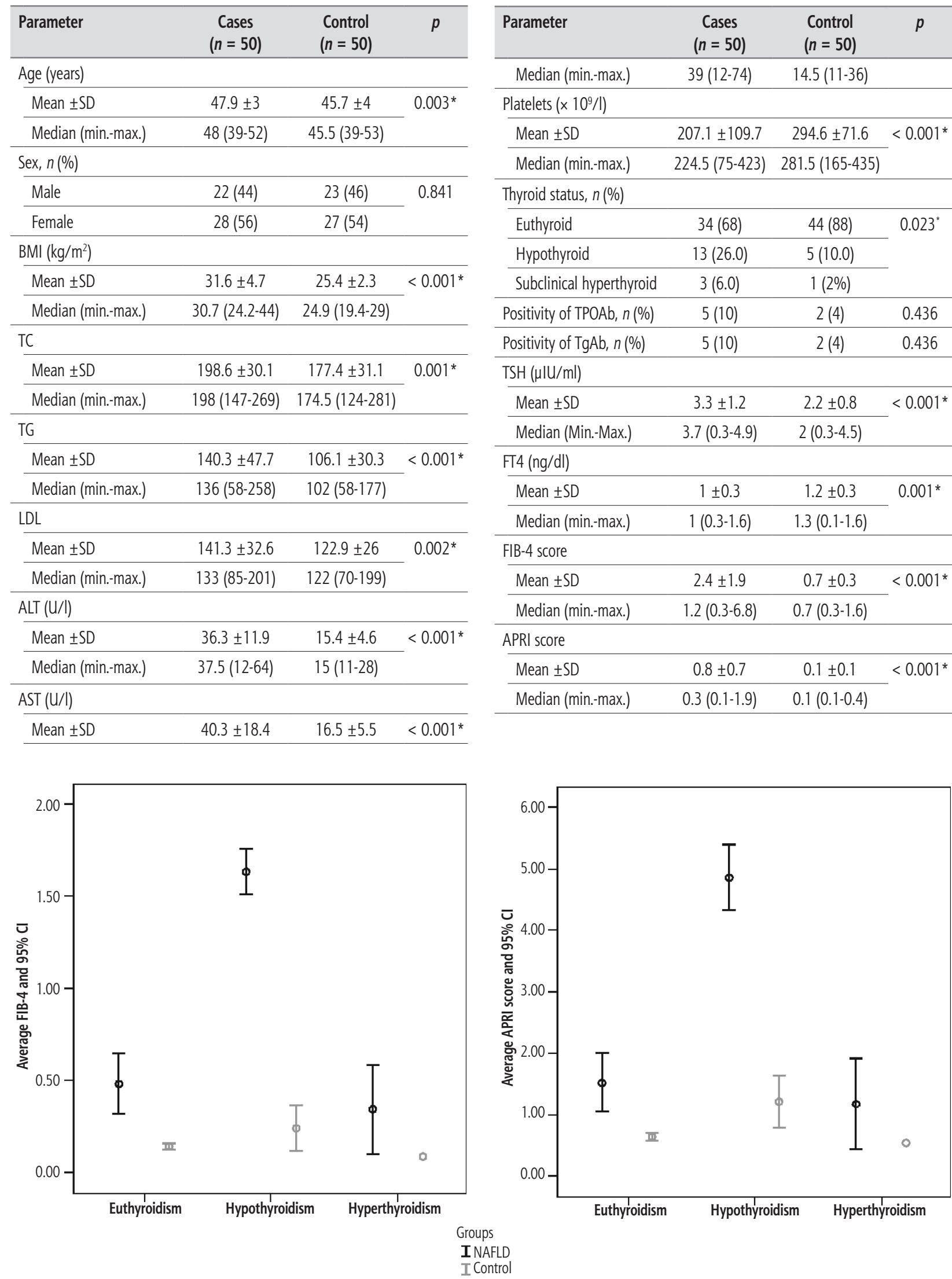

Fig. 1. Comparison of average APRI scores and FIB-4 score and its $95 \%$ confidence intervals between NAFLD cases and controls according to their thyroid status 

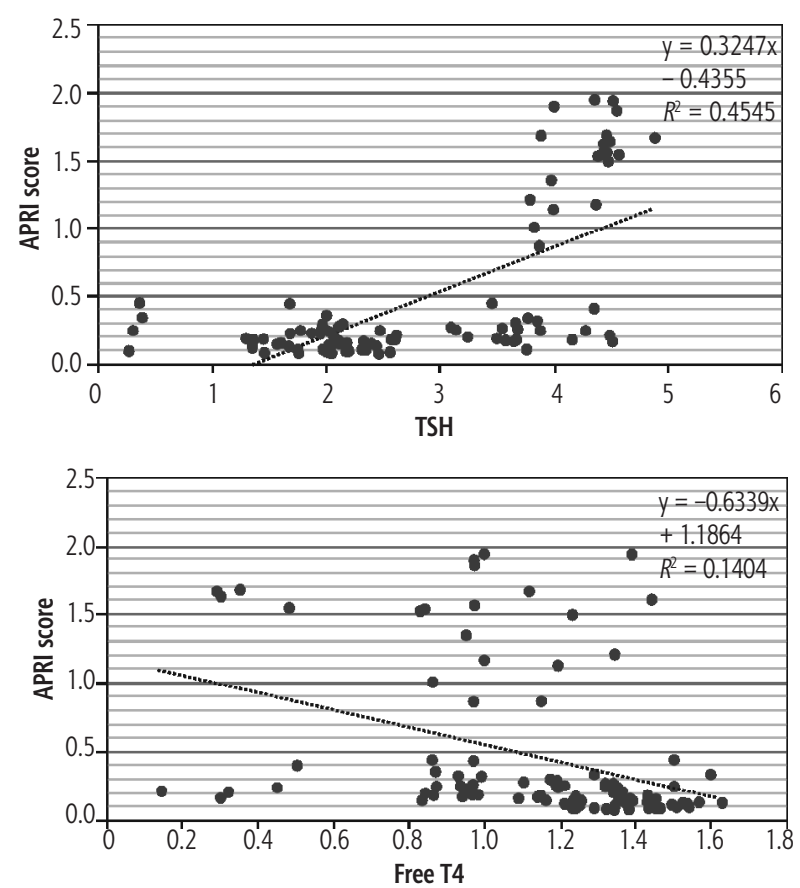

Fig. 2. Correlation between TSH and FT4 with APRI score and FIB-4 score

causes a decrease in energy expenditure, lipolysis, and gluconeogenesis, as well as an increase in weight and cholesterol levels. Hypothyroidism, as a result, causes hyperlipidemia, obesity, and insulin resistance, all of which are risk factors for metabolic syndrome associated with NAFLD. Thyroid hormones have been shown to promote hepatic lipogenesis and $\beta$-oxidation. As a result, they are associated with hepatic fat accumulation [1]. However, the link between NAFLD and thyroid function parameters is debatable. This might be attributable mostly to the variability of NAFLD diagnostic criteria, patient characteristics, and sample size. Another possible explanation is the possible differences in the criteria for diagnosing hypothyroidism and the measurement of thyroid hormones. In our study, the prevalence of hypothyroidism was significantly higher in NAFLD patients compared to controls

Table 2. Correlation between different parameters in case group $(n=50)$

\begin{tabular}{lcc}
\hline Parameter & APRI score & FIB-4 score \\
& $r$ & $r$ \\
\hline TSH $(\mu \mathrm{l} \mathrm{U} / \mathrm{ml})$ & $0.76^{*}$ & $0.73^{*}$ \\
\hline Cases & $0.66^{*}$ & $0.66^{*}$ \\
\hline Control & $0.88^{*}$ & $0.43^{*}$ \\
\hline FT4 $(\mathrm{ng} / \mathrm{dl})$ & $-0.39^{*}$ & $-0.38^{*}$ \\
\hline Cases & $-0.32^{*}$ & $-0.33^{*}$ \\
\hline Control & $-0.61^{*}$ & $-0.51^{*}$ \\
\hline
\end{tabular}

* Statistically significant at $p \leqslant 0.05, r-$ Pearson correlation coefficient
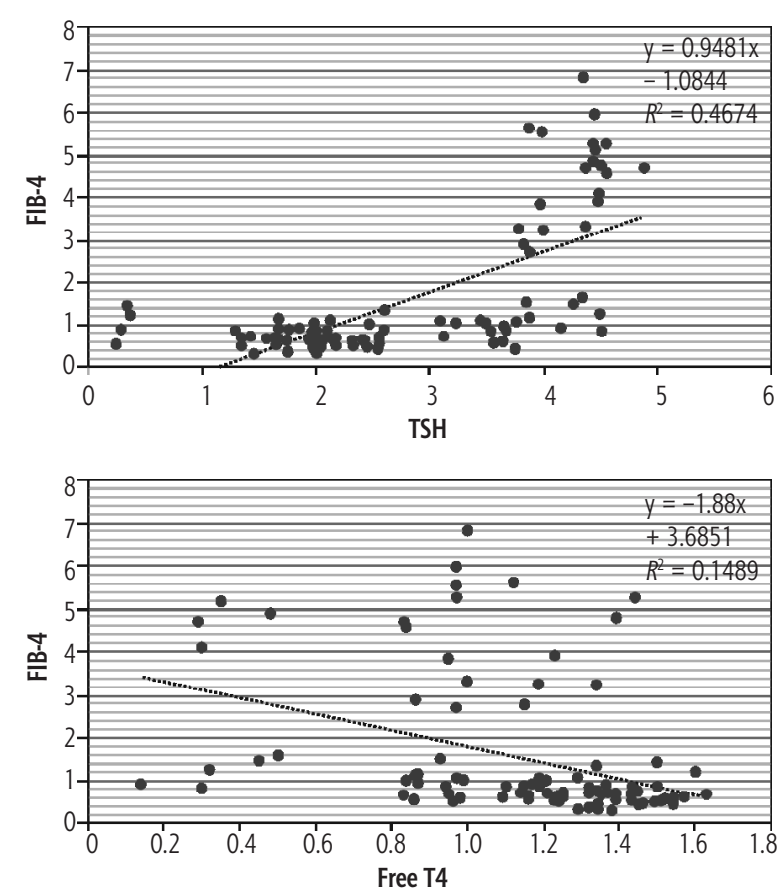

(about one quarter of patients with NAFLD had hypothyroidism compared to $10 \%$ of the control group). In a systematic review published in 2014, the authors found a prevalence of hypothyroidism in patients with NAFLD ranging from $15.2 \%$ to $36.3 \%$ [25], whereas the national estimated incidence of hypothyroidism in the US population was $3.7 \%$. In another study, hypothyroidism was shown to be more common in patients with biopsy-proven NAFLD than in matched controls ( $21 \%$ vs. $9.5 \%$, respectively) [26]. A new systematic review and meta-analysis of over 42000 individuals from 13 studies discovered a substantial link between hypothyroidism and NAFLD, giving solid epidemiological evidence for the risk of NAFLD in hypothyroidism, as compared to euthyroid people [27]. In contrast, Jaruvongvanich et al. reported that NAFLD is not linked to thyroid hormone levels or hypothyroidism [15]. The involvement of the thyroid hormone receptor in hepatic stellate cell activation has recently been proposed, although it is still unknown whether or how thyroid dysfunction accelerates the progression of NAFLD to steatohepatitis and, as a result, advanced fibrosis. Thyroid hormone replacement therapy lowers serum lipids significantly and has a positive impact on obesity. In individuals with hypothyroidism, levothyroxine given for 15 months improved serum transaminases and ultrasound diagnosed NAFLD [19]. After 4 months of low-dose levothyroxine treatment, individuals with NAFLD showed a decrease in hepatic fat content as assessed by magnetic resonance spectroscopy [28]. 
Even though we did not do a liver biopsy, the FIB-4 index is the most effective diagnostic modality in distinguishing individuals with advanced fibrosis. In our study, FIB-4 score and APRI score were significantly higher in patients with hypothyroidism compared to euthyroid patients. These results are comparable to a recent study which reported that patients with hypothyroidism had a substantially higher FIB-4 score than patients with euthyroidism [1]. TSH may play a role in the development of fibrosis in the liver. Thyroid hormones may have an influence on adiponectin regulation, which might help to limit the progression of fibrosis $[1,25]$. As a result, L-thyroxine therapy in individuals with hypothyroidism may improve liver fibrosis. Hypothyroidism is an independent predictor of progressive fibrosis, according to Kim et al. [29].

In the current study, levels of thyroid antibodies did not show any difference between NAFLD cases and control subjects. Another study reported that $\mathrm{TgAb}$ levels were shown to have an independent negative relationship with NAFLD. This study also reported that the prevalence of NAFLD decreased significantly with the increase of serum thyroid antibodies, indicating that $\mathrm{TgAb}$ may be a protective factor for NAFLD [20]. Another study, performed by Chen et al. [30], discovered a link between thyroid antibodies and the incidence of NAFLD. The disparity in results might be attributable to differences in the study population, sample size and diagnostic criteria. As a result, there is not only a lack of consistent findings in the link between thyroid autoantibody positivity and the incidence of NAFLD, but also a lack of knowledge of the different functions of $\mathrm{TPOAb}$ and $\mathrm{TgAb}$, which necessitates further research.

Our study has some limitations. First, the sample size was small. Second, because NAFLD was diagnosed by ultrasonography, the identification of mild steatosis was limited. Performing liver biopsy is recommended.

\section{Conclusions}

Hypothyroidism is more prevalent among patients with NAFLD compared to control subjects and high levels of TSH with low FT4 might be a risk factor for NAFLD and may impact the development of liver fibrosis. The role of thyroid autoimmunity in NAFLD needs further assessment. Until more data are available, NAFLD patients should be monitored by yearly TSH and FT4 testing.

\section{Funding}

This research was funded by the Deanship of Scientific Research at Princess Nourah bint Abdulrahman
University through the Fast-track Research Funding Program.

\section{Disclosure}

The authors declare no conflict of interest.

\section{References}

1. Tahara K, Akahane T, Namisaki T, et al. Thyroid-stimulating hormone is an independent risk factor of non-alcoholic fatty liver disease. JGH Open 2020; 4: 400-404.

2. Parikh P, Phadke A, Sawant P. Prevalence of hypothyroidism in nonalcoholic fatty liver disease in patients attending a tertiary hospital in western India. Indian J Gastroenterol 2015; 34: 169-173.

3. Eslam M, Valenti L, Romeo S. Genetics and epigenetics of NAFLD and NASH: Clinical impact. J Hepatol 2018; 68: 268-279.

4. Guo Z, Li M, Han B, et al. Association of non-alcoholic fatty liver disease with thyroid function: A systematic review and meta-analysis. Dig Liver Dis 2018; 50: 1153-1162.

5. Janovsky CCPS, Cesena FH, Valente VAT, et al. Association between thyroid-stimulating hormone levels and non-alcoholic fatty liver disease is not independent from metabolic syndrome criteria. Eur Thyroid J 2018; 7: 302-307.

6. Wang B, Wang B, Yang Y, et al. Thyroid function and non-alcoholic fatty liver disease in hyperthyroidism patients. BMC Endocr Disord 2021; 21: 27.

7. Mantovani A, Byrne CD, Bonora E, et al. Nonalcoholic fatty liver disease and risk of incident type 2 diabetes: a meta-analysis. Diabetes Care 2018; 41: 372-382.

8. Armstrong MJ, Adams LA, Canbay A, et al. Extrahepatic complications of nonalcoholic fatty liver disease. Hepatology 2014; 59: 1174-1197.

9. Eshraghian A, Jahromi AH. Non-alcoholic fatty liver disease and thyroid dysfunction: a systematic review. World J Gastroenterol 2014; 20: 8102-8109.

10. Marino L, Jornayvaz FR. Endocrine causes of nonalcoholic fatty liver disease. World J Gastroenterol 2015; 21: 11053-11076.

11. Ludwig U, Holzner D, Denzer C, et al. Subclinical and clinical hypothyroidism and non-alcoholic fatty liver disease: A cross-sectional study of a random population sample aged 18 to 65 years. BMC Endocr Disord 2015; 15: 1-7.

12. Gnocchi D, Massimi M, Alisi A, et al. Effect of fructose and 3,5-diiodothyronine (3,5-T2) on lipid accumulation and insulin signalling in non-alcoholic fatty liver disease (NAFLD)-like rat primary hepatocytes. Horm Metab Res 2014; 46: 333-340.

13. Cable E, Finn P, Stebbins J, et al. Reduction of hepatic steatosis in rats and mice after treatment with a liver-targeted thyroid hormone receptor agonist. Wiley Online Libr 2008; 49: 407-417.

14. Ludwig U, Holzner D, Denzer C, et al. Subclinical and clinical hypothyroidism and non-alcoholic fatty liver disease: a cross-sectional study of a random population sample aged 18 to 65 years. BMC Endocr Disord 2015; 15: 41.

15. Jaruvongvanich V, Sanguankeo A, Upala S. Nonalcoholic fatty liver disease is not associated with thyroid hormone levels and hypothyroidism: a systematic review and meta-analysis. Eur Thyroid J 2017; 6: 208-215.

16. Bano A, Chaker L, Plompen EPC, et al. Thyroid function and the risk of nonalcoholic fatty liver disease: The Rotterdam study. J Clin Endocrinol Metab 2016; 101: 3204-3211.

17. Webb M, Yeshua H, Zelber-Sagi S, et al. Diagnostic value of a computerized hepatorenal index for sonographic quantification of liver steatosis. Am Roentgen Ray Soc 2009; 192: 909-914. 
18. van den Berg E, van Tienhoven-Wind L, Amini M, et al. Higher free triiodothyronine is associated with non-alcoholic fatty liver disease in euthyroid subjects: the Lifelines Cohort Study. Metabolism 2017; 67: 62-71.

19. Gökmen FY, Ahbab S, Ataoğlu HE, et al. FT3/FT4 ratio predicts non-alcoholic fatty liver disease independent of metabolic parameters in patients with euthyroidism and hypothyroidism. Clinics (Sao Paulo) 2016; 71: 221-225.

20. Zhang X, Li R, Chen Y, et al. The Role of thyroid hormones and autoantibodies in metabolic dysfunction associated fatty liver disease: $\operatorname{TgAb}$ may be a potential protective factor. Front Endocrinol (Lausanne) 2020; 11: 1-11.

21. Silveira MG, Mendes FD, Diehl NN, et al. Thyroid dysfunction in primary biliary cirrhosis, primary sclerosing cholangitis and non-alcoholic fatty liver disease. Liver Int 2009; 29: 1094-1100.

22. Assem M, Fawzi M, Ibrahim A, et al. Thyroid dysfunction and insulin resistance in patients with nonalcoholic fatty liver disease. Egypt J Intern Med 2018; 30: 97.

23. Tietz Textbook of Clinical Chemistry and Molecular Diagnostics. E-Book. Burtis CA, Ashwood ER, Bruns DE - Google Books [Internet] (cited 2021 Aug 23).

24. Yen YH, Kuo FY, Kee KM, et al. APRI and FIB-4 in the evaluation of liver fibrosis in chronic hepatitis $\mathrm{C}$ patients stratified by AST level. PLoS One 2018; 13: e0199760.

25. Eshraghian A, Jahromi AH. Non-alcoholic fatty liver disease and thyroid dysfunction: A systematic review. World J Gastroenterol 2014; 20: 8102-8109.

26. Pagadala MR, Zein CO, Dasarathy S, et al. Prevalence of hypothyroidism in nonalcoholic fatty liver disease. Dig Dis Sci 2012; 57: 528-534.

27. He W, An X, Li L, et al. Relationship between hypothyroidism and non-alcoholic fatty liver disease: a systematic review and meta-analysis. Front Endocrinol (Lausanne) 2017; 8: 335.

28. Singeap AM, Stanciu C, Huiban L, et al. Association between nonalcoholic fatty liver disease and endocrinopathies: clinical implications. Can J Gastroenterol Hepatol 2021; 2021: 6678142.

29. Kim D, Kim W, Joo S, et al. Subclinical hypothyroidism and low-normal thyroid function are associated with nonalcoholic steatohepatitis and fibrosis. Elsevier [Internet]. [cited 2021 Sep 4]; Available from: https://www.sciencedirect.com/science/article/ pii/S1542356517309783

30. Chen Y, Wang N, Chen Y, et al. The association of non-alcoholic fatty liver disease with thyroid peroxidase and thyroglobulin antibody: A new insight from SPECT-China study. Autoimmunity 2018; 51: 238-244. 